\title{
Foreword to the Special Section on Reconfigurable Computing
}

\author{
Steven Derrien ${ }^{1} \cdot$ Kubilay Atasu $^{2}$ • João M. P. Cardoso ${ }^{3} \cdot$ Juergen Becker $^{4}$
}

Published online: 24 March 2017

(C) Springer Science+Business Media New York 2017

In the past two decades, reconfigurable computing has evolved from a niche design technology to a mainstream computing technology, as testified by its use in datacenters and its availability in cloud computing infrastructures. The main reason is that thanks to its flexibility, reconfigurable hardware can serve many different application domains, from arithmeticintensive digital signal-processing applications to narrow niche applications, such as enumerative combinatorics.

This special section of the Springer's Journal of Signal Processing Systems (JSPS) illustrates the diversity in the field of reconfigurable computing with its five papers describing application-specific FPGA accelerators that span a wide spectrum of applications. The five papers presented in this special section are briefly summarized below.

The first paper, "A Flexible, High-Performance FPGA Implementation of a Feed-Forward Equalizer for Optical Interconnects up to $112 \mathrm{~Gb} / \mathrm{s}$ " by Maragos et al.

Steven Derrien

steven.derrien@univ-rennes1.fr

Kubilay Atasu

kat@zurich.ibm.com

João M. P. Cardoso

jmpc@acm.org

Juergen Becker

juergen.becker@kit.edu

1 IRISA/INRIA, University of Rennes 1, Rennes, France

2 IBM Research, Zurich, Switzerland

3 University of Porto, Porto, Portugal

4 KIT, Karlsruhe, Germany (10.1007/s11265-016-1201-y), presents a highperformance FPGA-based architecture aimed at supporting optical interconnects in datacenters. Specifically, the work focuses on the problem of implementing the digital equalization stage, which is addressed through the use of a highly parallel implementation of a feed-forward equalizer (FFE). The results show that links up to 112 Gbps can be supported on state-ofthe-art FPGA devices.

The second paper, "An Exploration Framework for Efficient High-Level Synthesis of Support Vector Machines: Case Study on ECG Arrhythmia Detection for Xilinx Zynq SoC" by Tsoutsouras et al. (10.1007/s11265-017-1230-1), describes a design exploration framework for Support Vector Machine FPGA accelerators. Their approach is based on High Level Synthesis technology, and is validated on a an ECG analysis and Arrhythmia detection system. Their exploration is performed at two levels, the first one at the behavioral level to expose data- and instruction-level parallelism, and the second one at the architectural level, given the target platform memory system characteristics. The resulting accelerator has been implemented on a Zynq programmable SoC and authors show that the FPGA implementation can achieve a speedup up to $78 \times$.

The next paper, "RImCom: Raster-order Image Compressor for Embedded Video Applications" by Ugurdag et al. (10.1007/s11265-016-1211-9), presents a hardware solution that enables real-time compression of raster-order video streams. The approach delivers compression factors of $66 \%$ in lossless and lossy modes, and is the the first approach to reach $60 \mathrm{fps}$ at Full HD with rate control. Thanks to the proposed approach, on-the-fly compression/decompression can be used for processing HD video streams, thus lowering the pressure on the memory system and leading to reductions in system cost, electromagnetic radiation and power consumption. 
In their paper "Dual-Data Rate Transpose-Memory Architecture Improves the Performance, Power and Area of Signal-Processing Systems", El-Hadedy et al. (10.1007/s11265-016-1199-1) propose a novel registerbased transpose memory architecture, in which the use of dual-edge clocked registers is one of the main contributions. This approach allows the circuit throughput to be doubled and improves the clock speed by eliminating the need for combinational logic. When applied in the context of 2D-DCT and 2D-IDCT blocks, the proposed approach brings a threefold speed-up while consuming $20 \%$ less area than previous work.

The fifth and the final paper, "Putting queens in carry chains, No. 27" by Thomas Preußer et al. (10.1007/s11265-016-1176-8), illustrates how FPGAs can also be used to help solve combinatorial problems. Their work, which is part of an open-source effort, aims at accelerating the search of solutions to a previously unsolved problem instance of the well-known N-Queens Puzzle. Their article describes how they partition the exploration space to enable efficient parallelization, and details how they could take advantage of the bit-level structure of FPGAs to solve this problem. An in-depth discussion of current results and a projection on the remaining run time to solve the problem are also provided in the paper.

Together, the five papers in this special section clearly demonstrates the relevance of reconfigurable computing for a wide range of application that arise in today's computing landscape.

We would like to acknowledge the support of the Journal of Signal Processing Systems Editor in Chief, the contributions from the authors of the papers, and the help of the reviewers which was very appreciated and helped to improve the final quality of the papers.

We hope you enjoy reading the papers included in this special section.

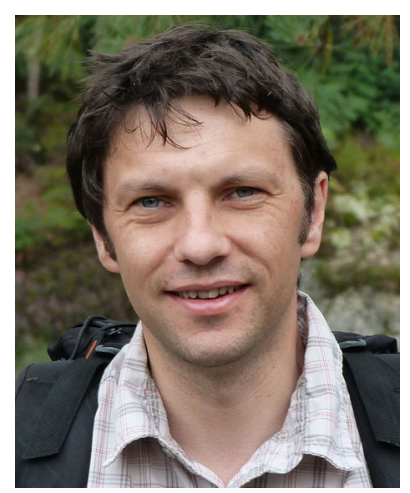

Steven Derrien obtained his $\mathrm{PhD}$ from University of Rennes 1 in 2003, where he is currently Full Professor at the Computer Science department, and a research member of the CAIRN group at IRISA and INRIA. His research interests include High-Level Synthesis, loop parallelization, and reconfigurable systems design.

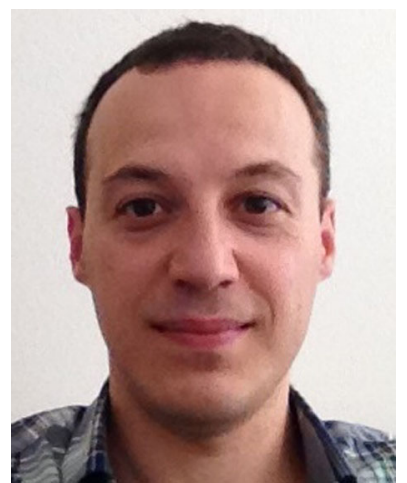

Kubilay Atasu received his Ph.D. degree in Computer Engineering from Bogazici University, Istanbul in 2008 and joined IBM Research - Zurich in the same year. From 2002 to 2003, he was with Swiss Federal Institute of Technology Lausanne (EPFL). From 2005 to 2008, he was with Imperial College London. His research interests include parallel computer architectures and algorithms, design of hardware accelerators, and use of hardware/software co-design and reconfigurable computing techniques to improve the computational efficiency, especially of Big Data applications. Kubilay was the recipient of a best paper award at the Design Automation Conference (DAC) in 2003, and at the IEEE International Conference on Application-specific Systems, Architectures and Processors (ASAP) in 2008. He was a program co-chair of ASAP 2013, a general co-chair of ASAP 2014, and a program co-chair of the 10th HiPEAC Workshop on Reconfigurable Computing. He served in the program committees of ASAP, FPL, FPT DATE, ICPP, and CF conferences, and is a member of the Editorial Board of Springer's Journal of Signal Processing Systems (JSPS). 


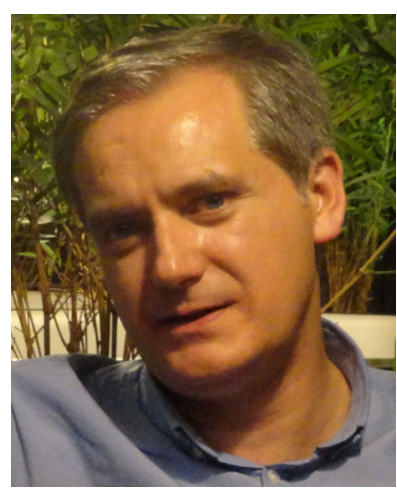

João M. P. Cardoso received his $\mathrm{PhD}$ degree in Electrical and Computer Engineering from the IST/UTL (Technical University of Lisbon), Lisbon, Portugal in 2001. He is currently Full Professor at the Department of Informatics Eng., Faculty of Eng. of the University of Porto, Porto, Portugal, and a research member of INESC TEC. Before, he was with the IST/UTL (2006-2008), a senior researcher at INESC-ID (2001-2009), and with the University of Algarve (19932006). In 2001/2002, he worked for PACT XPP Technologies, Inc., Munich, Germany. He has been involved in the organization and served as a Program Committee member for many international conferences. For example, he was general Co-Chair of IEEE/IFIP EUC'2015 and IEEE CSE'2015, General Chair of FPL'2013, General Co-Chair of ARC'2014 and ARC'2006, Program Co-Chair of ARCS'2016, DASIP'2014, and RAW'2010. He has (co-)authored over 150 scientific publications on subjects related to compilers, embedded systems, and reconfigurable computing. He has coordinated a number of research projects. He is a senior member of IEEE, a member of IEEE Computer Society, and a senior member of ACM. His research interests include compilation techniques, domain-specific languages, reconfigurable computing, application-specific architectures, and high-performance computing with a particular emphasis in embedded computing.

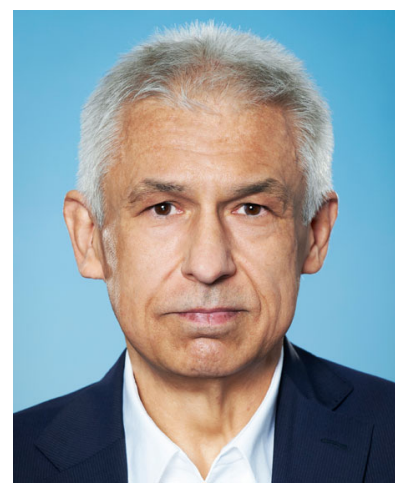

Jürgen Becker received the Diploma and Ph.D. (Dr.-Ing.) degree from Technical University Kaiserslautern, Germany. He is a full professor for embedded electronic systems and Head of the Institute for Information Processing Technologies (ITIV) at the Karlsruhe Institute of Technology (KIT). From 20052009 he has been appointed as Vice President for Education at Universitaet Karlsruhe (TH) and Chief Higher Education Officer (CHEO) at KIT from 2009-2012.

Since 2012 till 2014 he served as Secretary General of CLUSTER, an association of 12 leading technical universities in Europe. In 2013 Prof. Becker received the Honorary Doctor award (Dr. h.c.) from Technical University Budapest (Hungary). His research interests include Hardware/Software Systems-on-Chip (SoC), Cyber-Physical Systems (CPS), Heterogenous Multicore (MC) Architectures, Reconfigurable Computing, Fast Data Acquisition, Filtering and Storage for Semiconductor Detectors. He authored more than 400 papers in international journals and conferences. 